Peter Kastberg*

\title{
Die Vertextung von Termini als Ausweis textueller Strategien in technischen Textsorten
}

\begin{abstract}
In this paper I argue that the textualization of terms in technical texts - be it as part of a procedural condensation or expansion - is determined by the author's textual and text generic strategy in appreciation of the knowledge level and knowledge interest of his addressee rather than the technical matter conveyed in the text.
\end{abstract}

\section{Zum Stellenwert des Adressaten beim Produzieren technischer Texte}

Noch zu Anfang dieses Jahrzehnts war zu lesen:

"Die Güte [...] einer fachsprachlichen Darstellung kann letztlich nur danach beurteilt werden, in welchem Grade es dem Verfasser gelungen ist, seine Äußerungen dem Sachverhalt und dem Fachgebiet angemessen zu gestalten."

(Reinhardt et al. 1992: 143)

Nach dieser Auffassung ist das Qualitätskriterium zur Beurteilung, ob ein Fachtext als ein gelungener anzusehen ist oder nicht, von der technischen Materie (das Stichwort ist 'Sachverhalt') und dessen Strukturierung in und von der Fachgemeinde (das Stichwort ist 'Fachgebiet') quasi schon vorgegeben. Die Aufgabe des Fachtextproduzenten bestünde folglich darin, seinen Text diesen Gegebenheiten als geltender Vertextungsnorm ${ }^{1}$ anzupassen. Ich werde natürlich keineswegs behaupten, daß die technische Materie für das Produzieren technischer Texte belanglos wäre. Aber als Norm für das Produzieren von techni-

1 Norm hier im Sinne von Chestermann (1998: 12).

* Peter Kastberg

Tysk Institut

Handelshøjskolen $i$ Århus

Fugelsangs Allé 4

DK-8210 Århus $V$ 
schen Texten erscheint sie einfach unzulänglich. Denn außer einem Inhaltskriterium weist sie keine Kommunikationsparameter ${ }^{2}$ auf, wie z.B.: an wen wendet sich der Verfasser mit seinem Text, in welcher Situation und zu welchem Zweck tut er das? Ich könnte z.B. sehr wohl einen angemessenen Fachtext in diesem Sinne verfaßt haben, wenn aber der intendierte Leser diesen Text nicht rezipieren und somit nicht verstehen kann, gelingt keine Kommunikation. Wenn es demnach die Intention des Verfassers ist, daß jemand seinen Text liest und versteht ${ }^{3}$, dann muß er diesem jemand einen vorgeschobenen Stellenwert in seinem Textproduktionsprozeß einräumen. Spätestens seit Akzentuierung des adressatengerechten Texteproduzierens durch die Arbeiten von Göpferich (1996 et passim) zum Technical Writing, kann der sich Verfasser eines Fachtextes mit der in seinem Text besprochenen Materie als Maßstab, an dem die "Güte" seines Textes zu messen wäre, nicht begnügen. Unter Adressat verstehe ich den Prototyp eines vom Verfasser intendierten Rezipienten seines Textes, der den Text zu dem vom Verfasser intendierten Zweck rezipiert ${ }^{4}$. Als Prototyp stellt sich der Adressat aus einer Reihe von als relevant angesehenen Merkmalen zusammen. Zum Kern dieser Merkmale, die sozio- und demographisch ausgerichtet sind, gehören das (Erkenntnis-)Interesse des Adressaten und daraus folgend die Funktion, die der Fachtext dem Adressaten erfüllen soll.

Wenn man davon ausgeht, daß kein Objekt eo ipso einen bestimmten Fachlichkeitsgrad aufweist (Kalverkämper 1996 et passim), dann ist das, was sich als mehr oder weniger fachlich ausweist, der Handlungszusammenhang und - im Sinne von Konkretisierung eines Handlungszusammenhangs - somit der Fachtext, in dem das Objekt seine Vertextung findet. In dieser wird ihm Fachlichkeit zugeteilt - und als Konsequenz dessen, das Fachsprache nur in Texten realisert ist ${ }^{5}$ : de facto zugeschrieben - . Demzufolge ist Fachlichkeit keine inhärente Qualität des Objektes, sondern eine Qualität der Vertextung des Objektes. So wird das Objekt, z.B. Kraftfahrzeug, je nach Adressat, Zweck und

2 Bzw. noch konkreter: Textualitätskriterien.

3 Dazu Eggins (1994: 2) “[...] language use is functional [und] that its function is to make meanings [...]".

4 Bzw. vom Auftraggeber oder vom Verfasser und vom Auftraggeber gemeinsam intendiert, wenn diese nicht identisch sind.

5 Vgl. hierzu grundlegend Göpferich (1995: 23ff). 
Situation anders - und auch fachlich anders - zu vertexten sein. Z.B. in einem Kinderbuch anders als in einem Lehrbuch für Ingenieurstudenten oder aber als in einem Fachlexikon. Damit bezieht sich adressatengerechte Vertextung von Termini nicht nur auf Texte, sondern auch auf Textsorten. In der vorliegenden Studie wird auf eine Ebene der Vertextung technischer Texte fokussiert, und zwar auf die Ebene der Fachlexik. Auf dieser Ebene beschäftige ich mich mit der Vertextung von Termini im laufenden Fachtext. Die Leitfrage dieser Studie lautet demnach, ob der Produzent die von ihm eingesetzten Termini in einer auf seinen Adressaten ausgerichteten Weise in den Fachtext einbringt, und - als Anschlußfrage - gegebenenfalls welchen Anstoß dies der weiteren Forschung zur Erarbeitung von Fachtextsortenprofilen geben kann.

\subsection{Zur Vertextung von Termini}

In Konkretisierung der Keller'schen Annahme, “[...] der Einsatz sprachlicher Mittel ist Symptom des damit verfolgten Zwecks" (1995: 120-121), ist auch die Art der Vertextung von Termini als Symptom eines verfolgten Zwecks anzusehen. Den Rahmen dieser Zweck-MittelRelation gibt Zimmermann vor:

"[Jeder] Versuch der Erreichung von Zielen durch sprachliches Handeln ist im Prinzip strategisch. Strategie bedeutet, daß ein Handeln am möglichen Handeln ... des anderen orientiert ist, es antizipatorisch einplant"

(Zimmermann 1984:141 zitiert nach Heinemann/Viehweger 1991: 214).

Zum Begriff der Strategie gehören einerseits die "antizipatorische Planung" und andererseits das "Ziel". In textlinguistische Termini umgesetzt, heißt das, daß man bei der Ausarbeitung des Textplanes "antizipatorisch" Adressat-, Funktions- und Situationsfaktoren berücksichtigen soll, damit das erwünschte Kommunikationsziel erreicht wird. Plan und Ziel sind so gewissermaßen als Korrelate aufzufassen, indem der Plan vom erwünschten Ziel abhängig ist, zumal - umgekehrt - der Plan durch die Existenz eines Ziels überhaupt erst entwickelt wird. Diese Abhängigkeit ruft zwangsläufig eine Dynamik hervor, die sich darin zeigt, daß die Textproduzentenstrategie als Korrelat zur antizipierten Textadressatenstrategie aufgefaßt werden kann, eben weil die Produzentenstrategie die - oder eine - Adressatenstrategie "antizipato- 
risch einplant". Zum antizipatorischen Einplanen gehört noch eine Perspektivierung, denn als Ausprägung der korrelativen Dynamik, hat der Verfasser eine Erwartungs-Erwartung, d.h. Erwartungen gegenüber den Erwartungen seitens seines Adressaten zum Fachtext. Nähert man sich der Fachtextproduktion aus dieser Perspektive, geht mit dem adressatengerechten Produzieren von Texten die Textoptimierung einher, weil so Textoptimierung überhaupt erst in Bezug auf den Adressaten und seine (erwartbaren!) Erwartungen zum Fachtext einen Sinn hat. Die Vertextung von Termini ist demnach einem Gefüge strategischer und wenn auch abstrakt - dialogischer Überlegungen untergeordnet, die sich

“[...] als das Resultat einer Kette von - in der Regel bewußt ablaufenden - Auswahl- und Entscheidungsoperationen [zeigen], durch die Lösungsschritte und Mittel markiert werden zur Durchsetzung kommunikativer Ziele."

(Heinemann/Viehweger 1991: 214)

Wenn man "Auswahl- und Entscheidungsoperationen" auf die Einbringung von Termini in den laufenden Fachtext appliziert, stehen im Prinzip zwei Möglichkeiten zur Verfügung6. Entweder als alleinstehender Terminus oder als vertexteter Terminus (Beispiele vgl. unten). Im ersten Fall setzt der Verfasser voraus, daß der Terminus alleine ausreicht, um beim Adressaten die Wissensmenge zu evozieren, die der Terminus im Text vertritt. Der Terminus dient im Text in diesem Falle als Platzhalter für eine Wissensmenge, die der Leser selbst aus seinem Vorwissen abrufen soll. Im zweiten Fall wird umgekehrt angenommen, der Terminus könne beim Adressaten diese Wissensmenge eben nicht evozieren. Da ich mich in der vorliegenden Studie ausschließlich mit der Vertextung von Termini beschäftige, werden alleinstehende Termini - in diesem Sinne - keine weitere Berücksichtigung finden ${ }^{7}$. Determiniert von der Linearität des Textes kann die Vertextung vor (1) oder nach (2) dem Terminus eingeführt werden:

(1) feinverteiltes, poröses Blei (Bleischwamm)

[Beleg (Brockhaus/Lexikon) $]^{8}$

6 Hierzu Kastberg (1997: 34ff).

7 Es ist anzumerken, daß sich die Entscheidung, einen Terminus nicht zu vertexten, natürlich auch auf textstrategische Überlegungen zurückzuführen ist.

8 Zum Korpus vgl. Abschnitt 2.1. 
(2) Die Energiedichte, also die pro Kilogramm Batteriegewicht beziehungsweise pro Liter Batterievolumen speicherbare Energiemenge

[Beleg (Dustmann/pop.wiss.Art.)]

Unter einer Vertextung verstehe ich eine abgeschlossene Menge an Informationen über den Terminus, die der Verfasser dem Terminus im laufenden Text gezielt zuordnet (im folgenden deshalb: Zuordnungskontext) und die ihrerseits den Terminus substituieren können. Als dessen Konsequenz haben Terminus und Zuordnungskontext im Text eine gegenseitige Stellvertreterfunktion. Damit sind sie paradigmatische Synonyme (in Anlehnung an Harweg 1979: 20), was diese Konstruktion von einer bloßen Determinans-Determinandum-Relation deutlich absetzt ${ }^{9}$. Dies illustriert, daß sich die Vertextung von Termini in diesem von mir eingeführten engen Sinne in einem relationalen Verhältnis zwischen Zuordnungskontext und Terminus zeigt. Ferner läßt sich aus den Beispielen ableiten, daß die erste Vertextung (1) eine Relation ist, bei der eine umfangreichere sprachliche Größe, und zwar der Zuordnungskontext feinverteiltes, poröses Blei, in der Linearität des Textes in eine graphemisch kleinere sprachliche Größe, und zwar den Terminus Bleischwamm, kondensiert wird. Im Beispiel (2) verläuft die Relation genau umgekehrt, hier wird der Terminus Energiedichte in seinen Zuordnungskontext, die pro Kilogramm Batteriegewicht beziehungsweise pro Liter Batterievolumen speicherbare Energiemenge, expandiert. Ob der Verfasser die erste Reihenfolge wählt - und somit die zweite abwählt - , oder ob er die zweite Reihenfolge wählt - und damit die erste abwählt - , d.h. ob er sich für den Prozeß des Kondensierens oder den Prozeß des Expandierens entscheidet, macht im strategischen Sinne seinen Spielraum aus. Folglich gelten die konkreten Manifestationen von Kondensationen und Expansionen im laufenden Text als taktische Einzelverfahren, die zur Stützung dieser oder jener Strategie beitragen (vgl. unten).

Daß der Zuordnungskontext dem Definitionstext der Terminologie ähnlich ist, zeigt sich in der - letztendlich - abstrakt semiotischen Zweiseitigkeit der Relation, da der Zuordnungskontext sich durch den Terminus vertreten läßt, wobei - umgekehrt - der Terminus für den $\mathrm{Zu}$ -

9 Eben an diesem Punkt setzt sich der von mir vorgeschlagene Zugang von vorhergehenden (z.B. Kalverkämper 1987) in entscheidender Weise ab. 
ordnungskontext stehen kann. Im semiotischen Sinne gilt so der Zuordnungskontext als Zeicheninhalt für den Terminus, der seinerseits als Zeichenausdruck für den Zuordnungskontext gilt. Wenn sich aber dieses semiotische Verhältnis im laufenden Text realisiert, dann ist es ein aktuelles und somit prinzipiell ein einzeltextgebundenes semiotisches Verhältnis ${ }^{10}$.

An obigem Beispiel (1) läßt sich ein aktuelles, semiotisches Verhältnis zeigen, in dem als Zuordnungskontext feinverteiltes, poröses Blei fungiert, während Bleischwamm als kondensiertes Korrelat dient. In der Linearität des Textes wird dem Adressaten so zunächst ein 'Inhalt' erklärt, für den er im nachhinein einen 'Ausdruck' zur Verfügung gestellt bekommt. Wenn also im Textverlauf der Zuordnungskontext vor dem Terminus steht, kann die Operation des Kondensierens im laufenden Text als eine onomasiologische Operation charakterisiert werden. Dadurch kann jetzt ein wesentliches Charakteristikum der prozeduralen Kondensation gezeigt werden, und zwar ihre Benennungsfunktion nach dem Muster:

$\left(1^{\mathrm{i}}\right)$ feinverteiltes, poröses Blei [nennt man] Bleischwamm

Als authentisches Beispiel dafür, daß diese Benennungsfunktion im laufenden Text durchaus auch formuliert wird, sei angeführt:

(3) Die negative Elektrode gibt Ionen ab. Man nennt sie Katode

[Beleg (Fachkunde Elektrotechnik/Lehrbuch)]

Wenn also im Zuge der prozeduralen Kondensation ein Zuordnungskontext mit einem Terminus versehen wird und in dieser Weise benannt wird, dann ist dies eine Terminologisierung im laufenden Fachtext. Für diese Studie sind solche Beispiele onomasiologischer Operationen dem Korpus entnommen worden und in die Belegsammlung eingegangen, in der sie nach dem Benennungsmuster Definiens > Definitor > Definiendum zusammengestellt worden sind. Dieses Muster versteht sich als eine Konkretisierung im Sinne von Versprachlichung der onomasiologischen Operation. Mit diesem Benennungsmuster ist dem Analysein-

$10 \mathrm{Da}$ die Qualitäten aktuell und einzeltextgebunden der Vertextung eigen sind, zeigt sich darin, daß der Zuordnungskontext, den der Produzent seinem Adressaten im laufenden Gesamttext zur Verfügung stellt, nicht oder nur sehr selten Wort für Wort identisch ist mit dem von DIN oder anderen Normungsinstitutionen vorgeschriebenen normierten Definitionstext. 
strumentarium gleichzeitig eine Verifikations- bzw. Falsifikationsprobe angeschlossen worden, die dafür sorgt, daß tatsächlich nur prozedurale Kondensationen herausfiltriert werden. Denn mit dem Einsatz des Operators 'Definitor' (prototypisch als 'Sein'-Prädikation oder formelhaft als ' = ' zum Ausdruck gebracht) können die beiden die Kondensation konstituierenden Größen auf ihre paradigmatische Synonymie (vgl. oben) hin geprüft werden, zumal der Definitor für die gegenseitige Substituierbarkeit der Größen birgt, denen er als Definitor dient.

Die Größen Definiens, Definitor und Definiendum, die ihrer Natur nach funktional festgelegt sind, können im Textverlauf natürlich materiell/formal auf unterschiedliche Art und Weise vertreten werden. Das Definiens wird von einer oder von mehreren dem Adressaten als bekannt vorausgesetzten lexikalischen Einheiten repräsentiert. Das Definiendum dagegen wird durch einen Terminus vertreten, den der Textproduzent als seinem Adressaten unbekannt voraussetzt. Zwischen den beiden befindet sich der Definitor, der durch orthographische, graphische oder meta-sprachliche Instruktionen im Text vertreten ist. $\mathrm{Zu}$ den orthographischen Instruktionen zähle ich: runde Klammern, die als Parenthesen fungieren, sowie Kommata, Gedankenstriche o.ä., die eine appositive Ergänzung oder einen Einschub eingrenzen. Als graphische Instruktionen werden Fettdruck, Kursive, Spatium und Pfeile jedweder Art aufgefaßt. Meta-sprachliche Instruktionen sind hier solche textkommentierenden Signale, die dem Adressaten kundgeben, daß er in der Linearität des Textes als nächstes einen benennenden Stellvertreter für das eben angeführte Definiens erwarten darf, etwa: d.h., nennt/bezeichnet man, dies/e/r/s.

Am oben angeführten Beispiel (1) erläutert, sieht das Analysemuster demnach folgendermaßen aus:

(1ii) [feinverteiltes, poröses Blei $]^{\text {Definiens }}>[()]^{\text {Definitor }}$,

[Bleischwamm] Definiendum

Die operationalisierbare Verifikation / Falsifikation dessen, daß es sich in diesem Fall tatsächlich um eine Kondensation handelt, besteht letztlich in der Substitutionsprobe, wobei

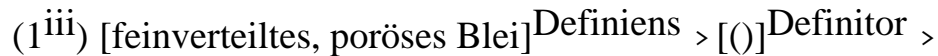
[Bleischwamm] Definiendum 


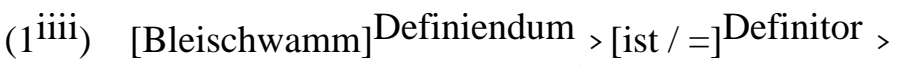
[feinverteiltes, poröses Blei] Definiens

transformiert werden kann, ohne daß dabei behauptet werden könnte, der Definitor sei als Operator ungültig.

Im Zuge des korrelativen Verhältnisses zwischen Kondensation und Expansion (Stichwort: gegenseitige Stellvertreterfunktion oben) realisiert sich die Expansion im laufenden Text als Spiegelbild der Kondensation. Wo der Zuordnungskontext bei der prozeduralen Kondensation in der Linearität des Textes vor dem Terminus steht, ist er bei der Expansion dem Terminus nachgestellt. In Abhängigkeit vom Perspektivenwechsel von der Kondensation auf die Expansion vollzieht sich ebenso eine Umkehrung von der onomasiologischen Operation des Kondensierens auf die semasiologische Operation des Expandierens. Dadurch begegnet der Adressat in der Linearität des Textes zunächst einem Terminus, einem 'Ausdruck', für den er im textuellen Nachhinein einen Zuordnungskontext, einen entsprechenden 'Inhalt' vom Textproduzenten zur Verfügung gestellt bekommt (vgl. Beispiel (2)). Der Einsatz solcher semasiologischen Operationen (und somit die Reihung der die Expansion konstituierenden Größen: Teminus > Zuordnungskontext) verstehe ich als Zeugnis der Textstrategie, daß der Produzent seinem Adressaten (Fach-)Ausdrücke im laufenden Text erklären möchte. Durch die semasiologische Betrachtungsweise wird ferner ein wesentliches Merkmal der prozeduralen Expansion deutlich, und zwar ihre Erklärungsfunktion nach dem Muster:

$\left(2^{\mathrm{i}}\right)$ Die Energiedichte [ bedeutet ] die pro Kilogramm Batteriegewicht beziehungsweise pro Liter Batterievolumen speicherbare Energiemenge

Ein Beispiel dafür, daß die Erklärungsfunktion auch explizit sprachlich im Text verankert sein kann, ist:

(4) Gute Leistungsaufnahme bedeutet eine möglichst verlustfreie Stromaufnahme an gegebener Ladespannung und schnelle Wiederaufladung

[Beleg (Krieg/Lehrbuch)]

Wenn also im Zuge der prozeduralen Expansion ein Terminus mit einem Zuordnungskontext versehen und in dieser Weise erklärt wird, 
dann ist dies eine Begriffsbildung im laufenden Fachtext. Da die Reihenfolge (Terminus >Zuordnungskontext) als Zeugnis einer Erklärungsstrategie aufgefaßt wird, kann man diese Produzentenstrategie nun damit exemplarisch erläutern, daß die Expansion ein taktisches Einzelverfahren ist, das über ein intendiertes Benennungslernen (i.e. das Erlernen der Benennung) zu einem intendierten Begriffslernen (i.e. das Erlernen des Zuordnungskontextes) im laufenden Text führt. Entsprechend obigem Perspektivenwechsel vollzieht sich beim Analyseraster sowie bei der Verifikations- / Falsifikationsprobe ebenso ein Perspektivenwechsel, wobei solche Beispiele semasiologischer Operationen dem Korpus entnommen worden und in die Belegsammlung eingegangen, die nach dem Erklärungsmuster Definiendum >Definitor 'Definiens zusammengestellt worden sind ${ }^{11}$.

\section{Ergebnisse und Implikationen}

\subsection{Der Studie zugrundliegendes Textkorpus}

Das für diese Studie untersuchte Textkorpus besteht aus 200 Normseiten dänischen Musters. Dieses Korpus setzt sich aus Texten und Textauszügen aus vier verschiedenen Textsorten zusammen, und zwar (nach Göpferich 1995) Fachzeitschriftenartikel, Lehrbuch, populärwissenschaftlichem Artikel und Lexikon. Jede Textsorte ist mit 50 Seiten im Korpus vertreten, und in jeder Textsorte ist eine Reihe verschiedener Fächer vertreten ${ }^{12}$.

\subsection{Ergebnisse}

Die Applikation des Analyserasters auf das Textkorpus ergab folgende Resultate, die unten tabellarisch und nach Textsorte differenziert aufgestellt sind.

\footnotetext{
11 Als - nicht authentisches - Beispiẹ für ein für die Analyse aufbereitetes Beispiel einer prozeduralen Expansion wäre (1 $1^{\text {iiii }}$ ) anzuführen.

12 Das Korpus ist Teil einer Arbeit, die in Vorbereitung ist.
} 


\begin{tabular}{|c|c|c|c|c|}
\hline \multirow[t]{2}{*}{ Textsorten } & \multicolumn{2}{|c|}{$\begin{array}{l}\text { Kondensationen: } \\
\text { Terminologisierung als } \\
\text { Ausweis intendierter Be- } \\
\text { nennungsstrategie im lau- } \\
\text { fenden Fachtext }\end{array}$} & \multicolumn{2}{|c|}{$\begin{array}{l}\text { Expansionen: } \\
\text { Begriffsbildung als Aus- } \\
\text { weis intendierter Erklä- } \\
\text { rungsstrategie im laufen- } \\
\text { den Fachtext }\end{array}$} \\
\hline & $\begin{array}{l}\text { absolute } \\
\text { Zahl }\end{array}$ & Prozentsatz & $\begin{array}{l}\text { absolute } \\
\text { Zahl }\end{array}$ & Prozentsatz \\
\hline Fachzeitschriftenartikel & 5 & $\gg 6 \%$ & 0 & $=0 \%$ \\
\hline Lehrbuch & 23 & $\gg 30 \%$ & 24 & $\gg 18 \%$ \\
\hline pop.wiss.Artikel & 7 & $\gg 9 \%$ & 13 & $» 10 \%$ \\
\hline Lexikon & 44 & $\gg 55 \%$ & 102 & $» 72 \%$ \\
\hline Gesamtzahl & 79 & $100 \%$ & 139 & $100 \%$ \\
\hline
\end{tabular}

In den folgenden Abschnitten werden die Ergebnisse der Analyse nach zwei Gesichtspunkten zu untersuchen sein. Zunächst nach der Art der Vertextung (Abschnitt 2.2.1. und 2.2.2.). Hier werden Kondensationen und Expansionen jeweils in ihrer Eigenschaft als Ausweis intendierter Benennungs- bzw. Erklärungsstrategie je nach Textsorte besprochen. Anschließend werden aus diesen Beobachtungen Aussagen zum etwaigen textsortenspezifischen Einsatz der beiden Vertextungsstrategien abgeleitet (Abschnitt 2.3.).

\subsubsection{Zur Vertextung von Termini als Ausweis intendierter Benennungsstrategie}

Die Erklärung dafür, daß diese Art der Vertextung (vgl. Beispiel 1) besonders im Lexikon (55\%) und im Lehrbuch (30\%) Anwendung findet, liegt vordergründig darin, daß die Produzenten solcher Texte didaktisch vorgehen. Denn außer Benennungsfunktion hat die Kondensation ebenso einen didaktischen Aspekt. Und zwar kann die Reihenfolge (Zuordnungskontext >Terminus) durchaus als Zeugnis einer Benennungsstrategie aufgefaßt werden, bei der die Kondensation zu einem taktischen Einzelverfahren abstrahiert wird, das über ein intendiertes Begriffslernen (i.e. das Erlernen des Zuordnungskontextes) zu einem intendierten Benennungslernen im laufenden Text führt. So gibt 
es denn auch etliche Beispiele, die diese Benennungsfunktion der Kondensationen im laufenden Text explizit zum Ausdruck bringen. Z.B. im Lehrbuch (vgl. Beispiel (2)) und im Lexikon:

(4) elektrochemische Stromquellen nennt man auch "Sekundärelemente"

[Beleg (Elektronik Lexikon/Lexikon)]

Es ergibt durchaus Sinn, daß im Lexikon und im Lehrbuch solche Kondensationen häufig vorkommen; denn in beiden Textsorten geht es hauptsächlich darum, daß ein (vom Produzenten angenommenes) Wissensdefizit des Adressaten gedeckt wird. Daß die Mehrzahl der Kondensationen in der Textsorte Lexikon zu verzeichnen ist, fügt sich - zumindest bei einer ersten Betrachtung - eigentlich sehr schön ins Bild, denn die Texsorte Lexikon wird sowohl von Benes \& (1973) als auch von Göpferich (1995) als eine 'kondensierte' Textsorte angesehen. Auf eine weitere Auseinandersetzung mit dieser Annahme sei aber vorerst verzichtet, ich verweise zugleich auf den Abschnitt 2.3., wo die Diskussion aufgenommen wird.

Besonders für die Textsorte Lehrbuch muß als relevant gelten, daß die Benennungsfunktion der Kondensation eine intendierte Terminologisierung im laufenden Text darstellt. Dem Adressaten wird so zur Interiorisierung von Termini im aktuellen Textverlauf verholfen, ohne daß er außerhalb des konkreten Textes - was einerseits zeit- und kräfteraubend und andererseits einem Lerner u.U. vielleicht nicht zumutbar wäre - nach den Inhalten dieser Termini suchen muß. Eine weitere didaktische Qualität einer solchen Terminologisierung im laufenden Text ist ferner, daß der Produzent im textuellen Nachhinein jetzt den Terminus alleine verwenden bzw. als wiederaufnehmendes Element einsetzen kann. Er kann nun sicher sein, daß der Adressat weiß vorausgesetzt natürlich dieser hat den vorhergehenden Text gelesen -, für welchen Zuordnungskontext der Terminus steht.

Die Erklärung dafür, daß die Vertextung von Termini als Kondensation in der Textsorte populärwissenschaftlicher Zeitschriftenartikel im Korpus verhältnismäßig schwach vertreten ist (9\%), steht noch aus. Dabei ist die Zahl der Belege in dieser Textsorte mit der Zahl derer in der Textsorte Fachzeitschriftenartikel fast identisch. Da ich vorwegnehme, daß die beiden Textsorten sehr verschiedene extra-linguistische Fachlichkeitsgrade aufweisen, kann die Erklärung für die annähernde 
Übereinstimmung der Belegzahlen nicht in der Übereinstimmung des Fachlichkeitsgrades liegen. Ich sehe sie vielmehr darin begründet, daß es nicht das Anliegen eines Produzenten von populärwissenschaftlichen Zeitschriftenartikeln ist, seinen Adressaten über ein intendiertes Begriffslernen (i.e. das Erlernen des Zuordnungskontextes) zu einem intendierten Terminologielernen (i.e. das Erlernen der Benennung) im laufenden Text zu bringen. Nicht - so nehme ich an - weil vermutet wird, der Adressat kenne diese Termini bereits, sondern weil angenommen wird, dem Adressaten sei nicht daran gelegen, diese Termini überhaupt erst kennenzulernen. Daraus ergibt sich, daß der Adressat, wenn er sich entschlossen hat, sich popularisierenden Darstellungen komplizierter Sachverhalte zuzuwenden, auch nicht erwartet, in diesen Darstellungen durch "schwierige" Fach-Wörter und Fach-Wendungen terminologisch herausgefordert $\mathrm{zu}$ werden. Eine Widerspiegelung der Neigung, Termini in dieser Textsorte nach Möglichkeit zu vermeiden und somit auch Terminologisierungen im laufenden Text zu vermeiden - , erkennt man daran, daß Termini, wenn sie in einer KondensationsRelation doch vorkommen, in 50 \% der Fälle durch eine Markierung hervorgehoben sind.:

(5) explosive Strahlungsausbrüche auf der Sonne, in der Fachsprache "Flares" genannt,

[Beleg (Schlege/pop.wiss.Art.)]

Und:

(6) Diese energiereichen Teilchen dringen hauptsächlich in polaren Gebieten in die Erdatmosphäre ein und verursachen damit ähnliche Störungen wie die Röntgenstrahlung (den sogenannten polaren Blackout)

[Beleg (Schlege/pop.wiss.Art.)]

Im Beispiel (5) ist das Signal der Fachsprachlichkeit markiert durch die Konstruktion in der Fachsprache genannt sowie durch die Anführungsstriche um den Terminus. Im Beispiel (6) erfolgt die Markierung durch das Signal den sogenannten. Im Vergleich zur sprachlich fixierten, reinen Benennungsfunktion (vgl. die Beispiele 3 und 4) ist der Signalwert in diesen Beispielen ein anderer. Denn wo die Benennungsfunktion lediglich feststellenden Charakter vom Typ "X nennt man Y" hat, ist eine Konstruktion vom Typ "X der sogenannte $\mathrm{Y}$ " (und somit 
die Wahl eben dieser Konstruktion) dazu da, dem Adressaten im voraus zu avisieren, er möge sich jetzt auf einen Terminus gefaßt machen. Die Wahl einer Markierung vom Typ "X der sogenannte Y", die das Fachliche am Terminus eher abtönt bzw. entschärft, soll dem Adressaten ferner ein zusätzliches Angebot signalisieren, das er in freier Entscheidung annehmen oder ablehnen kann, denn er hat die Wahl, einen neuen Terminus $\mathrm{zu}$ erlernen oder $\mathrm{zu}$ überlesen bzw. sofort wieder $\mathrm{zu}$ vergessen. Damit habe ich auch eine mögliche Antwort auf die Frage, warum Kondensationen in dieser Textsorte überhaupt vorkommen. Sie können als ein Angebot an den Adressaten vom Typ 'Laie' aufgefaßt werden, sich Teile eines fachmännischen Lexikons anzueignen.

Zur Erklärung dafür, daß es im Korpus in der Textsorte Fachzeitschriftenartikel nur fünf Belege dieses Typus gibt (6\%), kann zunächst negativ argumentiert werden, daß es dem Produzenten solcher Texte offenbar nicht darum geht, daß seine Adressaten über ein intendiertes Begriffslernen (i.e. das Erlernen des Zuordnungskontextes) zu einem intendierten Terminologielernen (i.e. das Erlernen der Benennung) kommen. Die Annahme liegt auf der Hand, daß der Produzent von einem Fachzeitschriftenartikel davon ausgeht, daß sein Adressat die Einbringung von Zuordnungskontexten nicht oder kaum nötig hat und diese sogar umgekehrt als redundant oder gar lesehemmend ansehen würde. Diese Annahme wird auch unterstützt durch die Beschaffenheit der vier von den insgesamt nur fünf Belegen dieses Typs im gesamten Textkorpus Fachzeitschriftenartikel, da sie deutliche Ähnlichkeiten untereinander aufweisen:

(7) die USV leitet im Normalbetrieb die Netzspannung an den Verbraucher weiter und wird erst bei Störungen aktiv (Konditioner) [Beleg (Gilke/fach.wiss.Art.)]

Im Vergleich zur explizit formulierten Benennungsfunktion z.B. in der Textsorte Lehrbuch (vgl. Beispiel 3) und zur Signalisierung der fachsprachlichen Zugehörigkeit in der Textsorte populärwissenschaftlicher Zeitschriftenartikel (vgl. Beispiel 5) tritt das Kondensat also in $80 \%$ der Belege dieser Textsorte als rein parenthetischer Zusatz auf. 


\subsubsection{Zur Vertextung von Termini als Ausweis intendierter Erklärungsstrategie}

Auf einige didaktische Implikationen der Erklärungsfunktion der prozeduralen Expansion (vgl. Beispiel 2 oben) hat schon Beier aufmerksam gemacht, indem er z.B. nachgewiesen hat, daß die Häufigkeit, mit der Definitionen - in der Terminologie dieser Studie funktional mit Zuordnungskontexten vergleichbar - im laufenden Text auftreten, vom (textsortengebundenen) Fachlichkeitsgrad des jeweiligen Fachtextes abhängt (1982: 23). Ihm zufolge enthalten Lehrbücher und Fachzeitschriften für Laien verhältnismäßig mehr Zuordnungskontexte als Texte eines höheren Fachlichkeitsgrades. Wenn Expansionen also in den Textsorten häufiger vorkommen, die einem Adressaten etwas erläutern wollen, so ergibt sich eine Erklärung für das sehr häufige Vorkommen von Expansionen in der Textsorte Lexikon (72\%) geradezu von selbst. Da die Nachschlagewerke, die ich untersucht habe, alle alphabetisch geordnete Makrostrukturen aufweisen (Bergenholtz/Tarp 1994: 240ff), liegt die jeweilige Zugriffsstruktur von vornherein als eine semasiologische fest. Dabei ist es nicht verwunderlich, wenn in dieser Textsorte eine verhältnismäßig hohe Anzahl von Expansionen zu finden ist. In Übertragung der Annahme Hausmanns, “[...] Wörterbücher sind immer didaktisch [...]" (1985: 121), auf meine eigene Annahme, daß Nachschlagewerke insgesamt inhärent didaktisch sind, kann eine semasiologische Zugriffsstruktur (und damit eine realisierte Expansions-Relation) in fachlichen Nachschlagewerken als Zeugnis für eine zugrundeliegende, didaktische Intention beim Einsatz von Expansionen gesehen werden.

$\mathrm{Daß}$ diese Art der Vertextung mit dem nächsthöchsten Prozentsatz in der Textsorte Lehrbuch im Korpus vorkommt (18\%), ist ebenso auf die didaktisch-strategischen Bemühungen des Produzenten zurückzuführen. Kalverkämper (1996: 143) akzentuiert eine solche Vertextung als Ausweis "[...] der partnerbezogenen, somit adressatengerichteten, also leserantizipierenden 'Erklärung' [...]', die er als bewußte Strategie zur Kommunikationssicherung wertet. Im Verhältnis zur Zahl der entsprechenden Kondensationen im laufenden Text kommen Expansionen im Korpus mehr als doppelt so oft vor. Wenn das Vorkommen von Expansionen also markant stärker ist, als das Vorkommen der entsprechenden Kondensationen, dann womöglich deswegen, weil einem Lerner die Relation der Expansion aus seinem Nachschlagen in Enzy- 
klopädien vertrauter ist. Indem ich davon ausgehe, daß der prototypische Adressat 'Lerner' mit dem Gebrauch von Nachschlagewerken vertraut ist, kann gleichzeitig eine Erklärung dafür angegeben werden, daß Expandate aus der Textsorte Lehrbuch der lexikographischen Definition sehr ähnlich sind:

(8) Elektrolyse ist das Zerlegen eines Stoffes durch Wandern und Entladen von Ionen mit elektrischem Strom.

[Beleg (Fachkunde Elektrotechnik/Lehrbuch)]

Der Unterschied zwischen diesen Expansionen und dem lexikographischen Muster (exemplarisch etwa: Lemma + lexikographische Definition) läßt sich - was die Beschaffenheit der Definition angeht - häufig auf das Vorhandensein oder Nicht-Vorhandensein eines sprachlich explizit fixierten Definitors ('ist' bzw. 'bedeutet') reduzieren. Denn gemäß "lexikographischer Formulierungskonvention" werden solche "[...] Relationsprädikate [...] ausgelassen" (Wolski 1997: 7) bzw. in Nachschlagewerken von einer durch das Lay-Out gegebenen Instruktion ersetzt. Im (alphabetisch geordneten) fachlichen Nachschlagewerk wird seiner Zugriffsstruktur wegen eine semasiologische Operation vom 'Ausdruck' zum 'Inhalt' verfolgt, wobei das Ergebnis dieses Nachschlagens das intendierte Erlernen von 'Inhalten', d.h. von sprachlich fixierten Begriffen ist. Im Lehrbuch dagegen wird eine solche semasiologische Operation mit Hilfe einer Expansion explizit in der Linearität des Textes formuliert. Da Expansionen intendierte Begriffsbildungen im laufenden Text verkörpern, ist es nicht verwunderlich, wenn Expansionen in der Textsorte populärwissenschaftlicher Zeitschriftenartikel doppelt so häufig vorkommen als Kondensationen. Denn da weder für den Produzenten noch für den Adressaten von Texten dieser Textsorte die Terminologisierung im laufenden Text im Vordergrund steht, kann gefolgert werden, daß es dem Produzenten von Texten dieser Textsorte um intendiertes Begriffslehren im laufenden Text, dem Adressaten wiederum um intendiertes Begriffslernen geht. Bemerkenswert ist ferner, daß die Expansionen in dieser Textsorte oft um eine Komponente erweitert werden. Und zwar um eine Komponente, die ich als eine Ausprägung der Intention des Produzenten, Verständnissicherung im laufenden Text zu gewährleisten, ansehe. Zur Erklärung soll folgendes Beispiel herangezogen werden: 
(9) die sogenannte Korona der Sonne. Diese dünne Gashülle erstreckt sich viele Millionen Kilometer in den kalten Weltraum und ist mit rund zwei Millionen Grad etwa zweihundertmal heißer als die Oberfläche der Sonne

[Beleg (Kayser/pop.wiss.Art.)]

In der Analyse sieht das Beispiel folgendermaßen aus:

(9i) [die sogenannte Korona der Sonne] Definiendum > [diese] Definitor > [ [dünne Gashülle] Informationsblock 1 [erstreckt sich viele Millionen Kilometer in den kalten Weltraum] Informationsblock 2 [ist mit rund zwei Millionen Grad etwa zweihundertmal heißer als die Oberfläche der Sonne] Informationsblock 3] Definiens.

Das Interessante daran ist weniger, daß das Definiendum von einem Mehrwortterminus vertreten wird und der Definitor von einer metakommunikativen Instruktion in Form von diese. Das Interessante ist vielmehr, daß das Definiens sich in drei Informationsblöcke mit z.T. sehr unterschiedlichen Aufgaben im Text grob aufgliedern läßt. Im ersten Block werden physikalische Angaben zum Material geboten, im zweiten physikalische Angaben zum Volumen gemacht und schließlich erfolgen im dritten physikalische Angaben zur Temperatur. Dieser letzte Block aber bietet dem Adressaten gleichzeitig einen Vergleich an: zweihundertmal heißer als die Oberfläche der Sonne. Wenn vielleicht nicht jeder Leser weiß, was er mit diesen drei physikalischen Angaben anfangen soll, so darf doch vorausgesetzt werden, daß fast jeder Mensch weiß, daß die Sonne sehr heiß ist; und wenn dann diese Korona zweihundertmal heißer ist als die Sonne, dann wird diese Temperatur zu einer (scheinbar) erfahrbaren Größe. Es wird m.a.W. im Zusatz zu den eher neutralen, faktengebundenen Informationen ein entscheidendes 'Mehr' an Informationen geboten, indem der Adressat vom Produzenten sozusagen gezwungen wird, auf einen (angenommenen) vertrauten Wissensblock ('die Sonne ist heiß') zurückzugreifen, damit er sich das volle Ausmaß der Hitze dieser Korona vorstellen kann ('die Korona ist aber zweihundertmal heißer'). Eine verständnissichernde Aufbereitung erfahren auch Expansionen, denen illustrative Beispiele unmittelbar nachgestellt werden, wie: 
(10) Ein Batterie-Kennwert ist die Spannung einer einzelnen Zelle, die nur eine negative und eine positive Elektrode enthält; sie beträgt beispielsweise beim Blei-Akkumulator etwa zwei Volt.

[Beleg (Hambitzer/Dreher/pop.wiss.Art.)]

Wenn ich in semiotischer Tradition davon ausgehe, daß die Bedeutung eines Zeichens, etwa der Termini in den Beispielen 9 und 10 oben, seine Übersetzung in ein anderes Zeichen ist, "in dem es voller entwickelt ist" (so Jakobson 1992: 482 in enger Anlehnung an Peirce), so folgere ich, daß der Produzent, indem er den Zuordnungskontext im Beispiel oben als ein Zeichen konzipiert hat, das "voller entwickelt ist" als der Terminus, dies absichtlich und deshalb getan hat, weil er sicher sein wollte, dem Adressaten ausreichende Informationen zur Verfügung gestellt zu haben, um von ihm verstanden zu werden.

Wie es bei verhältnismäßig vielen Kondensationen dieser Textsorte der Fall war, kommen auch bei den Expansionen Markierungen des Fachlichen öfter vor, und zwar ausschließlich in den Textsorten populärwissenschaftlicher Zeitschriftenartikel und Lexikon. Während bei Kondensationen mit einem dem Kondensat vorangestellten Signal dem Adressaten mitgeteilt wird, daß "jetzt ein Terminus kommt", kann bei Expansionen mit einem solchen Signal als Definitor dem Adressaten signalisiert werden: "Du hast zwar soeben einen Terminus gelesen, seine Erklärung / Bedeutung folgt aber sofort”. Vgl.:

(11) die Kapazität, also die entnehm- oder ladbare Strommenge [Beleg (Hambitzer/Dreher/pop.wiss.Art.)]

Mit dem Einsatz von Signalen dieser Art wird eine Abtönung des Fachlichen in den Texten der Textsorte populärwissenschaftlicher Zeitschriftenartikel insgesamt bewußt verfolgt. Als tendenziell ebenso typisch kann die Tatsache angesehen werden, daß eine Abtönung des Fachlichen im Lehrbuch weder bei den Kondensationen noch bei den Expansionen üblich ist (vgl. bedeutet im Beispiel 4 und ist im Beispiel 8, die rein feststellenden Charakter haben).

Die Textsorte Fachzeitschriftenartikel weist im gesamten Korpus keine Vertextungen von Termini dieser Art auf (dazu vgl. unten). 


\subsection{Zur textsortenspezifischen Vertextung von Termini}

Während ich in den vorhergehenden Abschnitten den Resultaten meiner Analyse einzeln nachgegengangen bin, werde ich mich zum Schluß damit befassen, die Einzelbeobachtungen zusammenzuführen und sie in einen größeren Rahmen je nach Textsortenzugehörigkeit der untersuchten Texte einzubetten.

Die Textsorte Fachzeitschriftenartikel zeichnet sich vordergründig dadurch aus, daß sie von Kondensationen sowie von Expansionen am sparsamsten Gebrauch macht. Auf den 50 Seiten, denen Texte und Teiltexte dieser Textsorte entnommen sind, gibt es nur 5 Vertextungen vom Typ "Zuordnungskontext >Terminus" und kein einziges Beispiel einer Vertextung vom Typ “Terminus > Zuordnungskontext". Die Gegenüberstellung der Belege in dieser Textsorte mit denen aus den anderen untersuchten Textsorten zeigt, daß das nachgewiesene weitgehende Fehlen von Kondensationen / Expansionen ein textsortenspezifisches Charakteristikum sein könnte. Ausgehend von der kommunikativen Konstellation dieser Textsorte (kurz: Fachmann - Fachmann), läßt sich auch eine Antwort dafür finden, warum Kondensationen häufiger vorkommen als Expansionen. Bei der symmetrischen Positionierung des Produzenten dieser Textsorte und seines Adressaten - sie sind 'Kollegen-im-Fach' - darf der Produzent davon ausgehen, daß sein Adressat sich im gemeinsamen Fach gut auskennt. Expansionen wären also deshalb hier weniger relevant, weil sie als Zeugnis einer Intention 'Begriffsbildung im laufenden Text' fungieren. Umgekehrt kann dem Einsatz von Kondensationen größere Relevanz zugesprochen werden, zumal dieser Zeugnis ist von einer Benennungsfunktion im Textverlauf, denn obwohl der Adressat sich - als Fachmann - (in grober Vereinfachung formuliert) mit den Begriffen und den Begriffssystemen seines Faches auskennt, kennt er kaum jede Benennung dieser Begriffe, oder aber es sind neue hinzugekommen bzw. es werden im aktuellen Text neue eingeführt, die er bislang nicht gekannt hat. Dafür spricht auch, daß Fachzeitschriftenartikel von Einzelfällen bzw. von Einzelausprägungen von Sachverhalten eines Faches handeln (im Gegensatz z.B. zum Lehrbuch, das von einem ganzen Fach bzw. einer ganzen Disziplin handelt).

In offensichtlichem Gegensatz zum Fachzeitschriftenartikel entfalten sich in der Textsorte Lehrbuch dagegen eine Fülle von Vertextungen von Termini. Es reicht nicht aus, eine typische Kommunikationskon- 
stellation dieser Textsorte (kurz) mit Fachmann - Laie anzugeben, denn der Begriff 'Laie' müßte um ein Merkmal 'Fachmann in spe' erweitert werden, zumal der Adressat ein Lerner ist und so - mit einem terminus technicus der Soziologie - Mitglied einer Instrumentalgruppe ist, deren übergeordnetes Ziel (vereinfacht gesagt) das Erlernen eines Faches ist. Somit geht es einem Adressaten dieses Typs nicht vordergründig darum, über Einzelfälle bzw. über Einzelausprägungen von Sachverhalten eines Faches, sondern über das Fach selbst informiert zu werden. $\mathrm{Zu}$ diesem Zweck werden in meinem Korpus - von einem Beleg abgesehen - Kondensationen und Expansionen quantitativ gleich eingesetzt. Wenn keine der beiden Strategien also in dieser Textsorte die vorherrschendere ist, kann als textsortenprofilierend angesehen werden, daß sich die Funktionen dieser beiden Strategien (Benennen einerseits und Erklären andererseits) in dieser Textsorte komplementieren, was in keiner der anderen von mir untersuchten Textsorten der Fall ist.

Im Unterschied zum Lehrbuch zeichnet sich der populärwissenschaftliche Zeitschriftenartikel dadurch aus, daß er fast doppelt so viele Expansionen als Kondensationen enthält. Beim Vergleich mit dem Fachzeitschriftenartikel ist zudem zu anzumerken, daß sich allmählich die Tendenz bemerkbar macht, daß mit fallendem Fachlichkeitsgrad die Zahl der Expansionen auf Kosten ihrer kondensatorischen Pendants zunimmt (hierzu weiter unten). Diese Tendenz spiegelt zudem wider, daß die Konstellation der Kommunikationspartner dieser Textsorte an einem wesentlichen Punkt von der oben besprochenen Kommunikationskonstellation $^{13}$ abweicht. So ist die Positionierung zwar noch asymmetrisch, der Produzent immer noch 'Fachmann' und der Adressat stets 'Laie'; dafür ist letzterer aber kein 'Fachmann in spe'14, und er wird somit auch nicht zur Rechenschaft gezogen für die Lektüre eines populärwissenschaftlichen Artikels, wie es dem Adressaten eines Lehrbuchs bei einer etwaigen Prüfung passieren kann. Auch muß in Betracht gezogen werden, daß die Zeitschrift / Zeitung, aus der der isolierte populärwissenschaftliche Zeitschriftenartikel stammt, sich aus einer Fülle von anderen Artikeln zusammensetzt, die wiederum intern weit weniger homogen sind, als es bei den anderen von mir untersuch-

13 Die sich wiederum selbstredend von der zuerst besprochenen Kommunikationskonstellation wesentlich absetzt.

14 Wenn doch, dann durch Zufall und somit nicht intendiert. 
ten Textsorten der Fall ist. Der Fachzeitschriftenartikel stammt aus einer Fachzeitschrift, dessen Texte ein "Kommunizieren über" - ein Fach eben - als gemeinsamen Nenner haben, das Lehrbuch ist ein Lehrbuch eben eines Faches, wogegen der populärwissenschaftliche Zeitschriftenartikel aus einer 'Textesammlung' (aus einer Zeitung, einer populärwissenschaftlichen Zeitschrift o.ä.) stammt, deren übrige Texte kein gemeinsames "Kommunizieren über" haben. Damit im Einklang stehen nun das verhältnismäßig geringe Auftreten von Kondensationen und umgekehrt das verhältnismäßig starke Auftreten von Expansionen, denn eine Terminologisierung im Textverlauf, die u.a. dazu dient, daß der Adressat die oder einige Termini eines Faches erlernen kann oder daß der Produzent im textuellen Nachhinein den Terminus nach Belieben als Platzhalter einsetzen kann, kann für diese Textsorte nicht primär in Frage kommen. Auch ist es unökonomisch, Termini in einem populärwissenchaftlichen Zeitschriftenartikel, der von einem bestimmten Fachgebiet handelt, einzuführen, wenn im nächsten populärwissenschaftlichen Zeitschriftenartikel derselben Zeitschrift, der ein anderes Fachgebiet behandelt, sowieso neue Terminologisierungen im laufenden Text durchgeführt werden müßten. Im Lehrbuch dagegen kann es umgekehrt als sehr ökonomisch gelten, Terminologisierungen in den laufenden Text einzubringen, da sich der Produzent ebenso wie der Adressat in einem ganzen Buch auf dieselben Terminologisierungen bei der Produktion bzw. Rezeption beziehen können.

Ebenso wie im populärwissenschaftlichen Zeitschriftenartikel treten auch in der Textsorte Lexikon eine Vielzahl an Kondensationen / Expansionen auf. Die Kommunikationspartner sind in diesem Fall - wie oben - Fachmann und Laie, im Unterschied zum Lehrbuch ist jedoch hier auch nicht die Rede von einem 'Fachmann in spe'. Im Gegensatz zu den drei anderen Textsorten zeichnet sich das Lexikon überdies dadurch aus, daß es üblicherweise nicht von A bis $\mathrm{Z}$ gelesen wird. Das Lexikon ist vielmehr so konzipiert, daß es Informationen in einer Weise anbietet, die es dem Adressaten - bauend auf sein (Erkenntnis)Interesse 'Informiert-zu-werden-über-einen-Einzelfall' - erlaubt, Informationen sehr gezielt zu suchen. Eine unmittelbare Gegenüberstellung der Belege der Kondensation und denen der Expansion führt zu einem auffallenden Ergebnis, denn während das Lexikon 44 Belege für Kondensationen aufweist, kommen mit 102 Belegen mehr als doppelt so viele Expansionen vor. Wenn also die Expansion vorherrschend ist, dann 
kann ich aus meiner Perspektive die geläufige Annahme nicht bestätigen, das Lexikon sei eine 'kondensierte' Textsorte, zumal sie offenbar vielmehr als eine von aktuellen Expansionen geprägte Textsorte angesehen werden kann. Auf dieser fachlexikalischen Ebene ist die Textsorte Lexikon ihres semasilogischen Aufbaus wegen in ihrer Konzipierung eher 'expansiv' ausgerichtet ${ }^{15}$. Ferner differenzieren meine empirischen Analysen die - oben kurz angesprochene - Behauptung Beiers:

"Die Häufigkeiten von Definitionen [i.e. Zuordnungskontexten] sind verschieden. An der Spitze liegen die Lehrbücher, gefolgt von den Fachzeitschriften und den Arbeiten für Laien."

(Beier 1982: 23)

Wenn ich Beier (und mit ihm Göpferich 1995: 384) auch zustimme, daß die Häufigkeit von Zuordnungskontexten eine maßgebliche Rolle bei der Typologisierung von Textsorten spielt, so zeigen obige Ausführungen doch, daß ich die Behauptung Beiers nuancieren und - von meinem Korpus ausgehend (!) - teilweise widerlegen kann. Zuordnungskontexte kommen in meinem Korpus im Lexikon nämlich viel häufiger vor als im Lehrbuch. Im Fachzeitschriftenartikel kommen sie fast nicht vor; im populärwissenschaftlichen Zeitschriftenartikel werden sie nahezu genauso sparsam verwendet wie im Fachzeitschriftenartikel. Die Frage nach dem Einsatz von Zuordnungskontexten verlangt m.a.W. eine differenziertere Antwort. Im Verhältnis z.B. zu den Beier'schen "Arbeiten für Laien", die - so muß ich als Leser annehmen - meinen populärwissenschaftlichen Zeitschriftenartikel und mein Lexikon umfassen, zeigt sich, daß diese beiden in bezug auf das Vorkommen von Zuordnungskontexten doch sehr unterschiedlich sind, weshalb sie unter einem so großmaschigen Sammelbegriff nicht aufgeführt werden können.

Es bietet sich als weitere Forschungsperspektive an, ein noch feinmaschigeres Textsortenraster $\mathrm{zu}$ applizieren, damit man zu differenzierteren Ergebnissen bezüglich des textsortenspezifischen Einsatzes von Zuordnungskontexten kommen kann. Ferner - und das ist mein Hauptanliegen an dieser Stelle - geht es aus den vorgelegten Analysen hervor, daß berücksichtigt werden muß, wie diese Zuordnungstexte in

15 Vgl. Kastberg (1997: 195ff). 
Relation zu ihren Termini im Text auftreten. Denn die Art der Vertextung - sei sie als Kondensation oder als Expansion - hat textsortenspezifischen Charakter, wobei sie am adressatengerechten Produzieren von technischen Texten ihren natürlichen Anschluß findet.

\section{Bibliographie}

Beier, Rudolf (1982). Zur Untersuchung der Fachsprache aus text- und pragmalinguistischer Sicht. In Fachsprachenforschung und -lehre. Gunter Narr Verlag. Tübingen (= Tübinger Beiträge zur Linguistik; 177). 15-27.

Benes, Eduard (1973). Die sprachliche Kondensation im heutigen deutschen Fachstil. In Sprache der Gegenwart. Linguistische Studien III. Teil 1. Pädagogischer Verlag Schwann. Düsseldorf. 40-50.

Bergenholtz, Henning; Sven Tarp (1994). Manual i fagleksikografi. Udarbejdelse af fagordbøger. Poblemer og løsningsforslag. Forlaget Systime. Herning.

Chestermann, Andrew (1998). On the Relevance of Translation Theory to Translator Training. In Germanic and Baltic Linguistic Studies and Translation. Vilnius. 6-15.

Eggins, Suzanne (1994). An Introduction to Systemic Functional Linguistics. Pinter Publishers. London.

Göpferich, Susanne (1995). Textsorten in Naturwissenschaft und Technik. Pragmatische Typologie - Kontrastierung - Translation. Gunter Narr Verlag. Tübingen (= Forum für Fachsprachen-Forschung; Band 27).

Göpferich, Susanne; Peter A. Schmidt (1996). Begriff und adressatengerechte Benennung: Die Terminologiekomponente beim Technical Writing. In Wissenschaftliche Grundlagen der Technischen Kommunikation. Gunter Narr Verlag. Tübingen (= Forum für Fachsprachen-Forschung; Band 32). 369-402.

Harweg, Roland (1979). Pronomina und Textkonstitution. Wilhelm Fink Verlag. München.

Hausmann, Franz Josef (1985). Kollokationen im deutschen Wörterbuch. Ein Beitrag zur Theorie des lexikographischen Beispiels. In Lexikographie und Grammatik. Akten des Essener Kolloquiums zur Grammatik im Wörterbuch. 28.-30.6.1984. Hrsg. von Bergenholtz/Mugdan. Max Niemeyer Verlag. Tübingen. 118-129.

Heinemann, Wolfgang; Dieter Viehweger (1991). Textlinguistik. Eine Einführung. Max Niemeyer Verlag. Tübingen (= Reihe Germanistische Linguistik; 115 Kollegbuch).

Jakobson, Roman (1992). Semiotik. Ausgewählte Texte 1919-1982. Hrsg. von Holenstein. Suhrkamp Verlag. Frankfurt am Main (=suhrkamp taschenbuch; 1007).

Kalverkämper, Hartwig (1987). Vom Terminus zum Text. In: Standpunkte der Fachsprachenforschung. Hrsg. von Sprissler. Gunter Narr Verlag. Tübingen (= Forum angewandte Linguistik; Band 11). 39-78. 
Kalverkämper, Hartwig (1996). Im Zentrum der Interessen: Fachkommunikation als Leitgröße. In Hermes Journal of Linguistics (= No 16). Handelshøjskolen i Århus. 117-176.

Kastberg, Peter (1997). Kondensation und Expansion in der Fachsprache der Technik. Handelshøjskolen i Århus. Århus[Präpublikation].

Reinhardt, Werner; Claus Köhler; Gunter Neubert (1992). Deutsche Fachsprache der Technik. Studien zu Sprache und Technik. Band 3. Hrsg. von Arntz / Wegner. Georg Olms Verlag. Hildesheim / Zürich / New York.

Wolski, Werner (1997). Textverdichtung und Textauflockerung im standardisierten Fachwörterbuchartikel. In Fachsprachen. Ein internationales Handbuch zur Fachsprachenforschung und Terminologiewissenschaft. Walter de Gruyter. Berlin / New York (= HSK - Handbücher zur Sprach- und Kommunikationswissenschaft; 14, erster Halbband). 1-17. [Präpublikation]. 


\title{
SPRACHGERMANISTIK IN SKANDINAVIEN III
}

\author{
Akten des IV. Nordischen Germanistentreffens \\ auf Schloß Sandbjerg \\ 5. bis 8 . Juni 1996
}

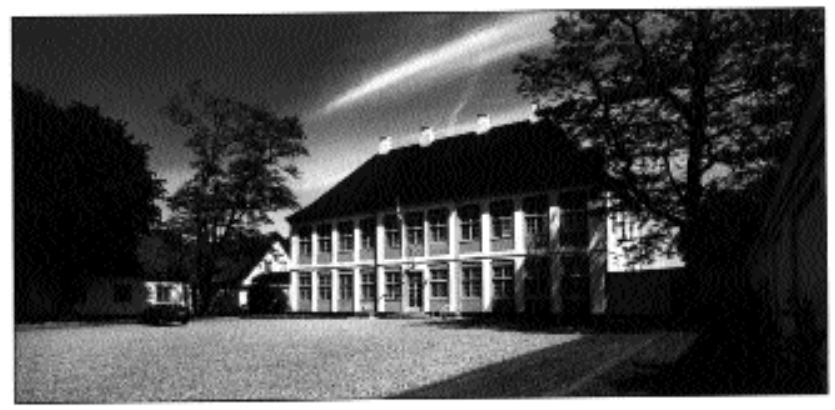

Herausgegeben von Harald Pors, Lisbeth Falster Jakobsen und

Flemming Talbo Stubkjær

\section{Pris: 215 DKK}

Kan bestilles på Handelshøjskolen i Århus

(e-mail: em@lng.hha.dk) 
